\title{
Theory of Negative-Refractive-Index Response of Double-Fishnet Structures
}

\author{
A. Mary, ${ }^{1}$ Sergio G. Rodrigo, ${ }^{2}$ F. J. Garcia-Vidal, ${ }^{1}, *$ and L. Martin-Moreno ${ }^{2}$ \\ ${ }^{1}$ Departamento de Fisica Teorica de la Materia Condensada, Universidad Autonoma de Madrid, E-28049 Madrid, Spain \\ ${ }^{2}$ Departamento de Fisica de la Materia Condensada-ICMA, Universidad de Zaragoza-CSIC, E-50009 Zaragoza, Spain
}

(Received 15 January 2008; revised manuscript received 3 August 2008; published 5 September 2008)

A theory is presented of the negative refractive index observed in the so-called double-fishnet structures. We find that the electrical response of these structures is dominated by the cutoff frequency of the hole waveguide whereas the resonant magnetic response is due to the excitation of gap surface plasmon polaritons propagating along the dielectric slab. Associated with this origin, we show how the negative refractive index in these metamaterials presents strong dispersion with the parallel momentum of the incident light.

DOI: 10.1103/PhysRevLett.101.103902

PACS numbers: 42.25.Bs, 73.20.Mf, 78.20.Ci

The phenomenon of extraordinary optical transmission (EOT) through periodic two-dimensional hole arrays (2DHA) drilled in metallic films has sparked considerable interest since the pioneering experiments [1]. It is now widely accepted that surface plasmon polaritons (SPPs) play a key role in the EOT phenomenon. Recently, Zhang and co-workers [2,3] have proposed and demonstrated a negative index metamaterial [4] working at near infrared frequencies with a design very similar to the structure showing EOT. This metamaterial is composed by a two-dimensional (2D) array of holes penetrating completely in a metal-dielectric-metal film stack. This double-fishnet (DF) structure has received a lot of attention for its negative refractive index (NRI) at visible [5] and near infrared frequencies [6,7]. Very recent studies have shown that this NRI metamaterial design also operates in the microwave frequency regime $[8,9]$. Metamaterials presenting NRI are expected to lead to important applications, with the perfect lens [10] a notable example.

In this Letter we present a theoretical study of the transmission properties of both 2DHA and DF structures. We demonstrate that the cutoff frequency of the hole waveguide marks the separation between positive and negative values for the effective electric permittivity. We also show that the negative magnetic permeability in the DF structure is due to the excitation of gap surface plasmon polaritons, leading to a high sensitivity of these magnetic resonances with the angle of incidence. Schematic pictures of the two systems under study (2DHA and DF) are shown in the insets of Figs. 1(a) and 2(a), respectively. The 2DHA structure is an infinite square array (period $d$ ) of rectangular holes of sides $a_{x}$ and $a_{y}$, perforated on a freestanding metallic film of thickness $h_{1}$. A DF structure can be described as three films (metal-dielectric-metal) drilled with a square array of rectangular holes, with $h_{m}$ and $h_{d}$ being the thickness of the metallic films and the dielectric layer, respectively. To further simplify the analysis without loosing generality, the dielectric between the two metal films is chosen to be vacuum or air. The geometrical parameters are $a_{x}=0.33 d, \quad h_{m}=0.05 d, \quad h_{d}=0.05 d, \quad$ and $\quad h_{1}=$ $2 h_{m}+h_{d}=0.15 d$. The long edge of holes $a_{y}$ will vary between $0.33 d$ and $0.98 d$. These values are representative of those used in the experimental samples showing NRI [6]. In our study, we analyze two very distinct frequency regimes. First, we consider that the metal behaves as a perfect electrical conductor (PEC), which is a very good approximation for metals at microwave or terahertz frequencies. When analyzing this limit, we will use $d$ as the unit length. We also present results for silver at optical and near infrared frequencies. In this case, we take the dielectric function of silver from [11] and we choose $d=$ $600 \mathrm{~nm}$. The structures are illuminated by a $p$-polarized plane wave (i.e., the in-plane component of the incident $E$ field pointing along the short edge of the holes). In order to analyze 2DHA and DF structures made of PEC material, we apply a theoretical formalism that is based on a modal expansion of the electromagnetic (EM) fields in the different spatial regions $[12,13]$. When analyzing perforated silver films at optical frequencies, we use instead the finite difference time domain (FDTD) method [14], which is virtually exact provided that a small enough mesh size is chosen to account for the rapid variations of the EM fields.

First, we revisit the transmission properties of a 2DHA made of rectangular holes. Figure 1(a) renders the corresponding normal incidence transmittance spectra as a function of wavelength $(\lambda)$ and $a_{y}$ for the PEC case. For small holes, two transmission peaks (leading to $100 \%$ transmission), whose spectral locations appear close to $d$, emerge in the spectrum. These two resonances correspond to the symmetric and antisymmetric combinations of the two surface EM modes associated with the two interfaces of the structure [12]. Note that, as the metal behaves as a PEC, these modes are not real SPPs but geometry-induced surface EM modes, the so-called spoof SPPs, as reported in [15]. As $a_{y}$ is increased, the extremely narrow peak associated with the antisymmetric combination remains unaltered at $\lambda \approx d$, but the symmetric one hybridizes strongly with a new resonance appearing at $\lambda_{C}=2 a_{y}$, the cutoff 


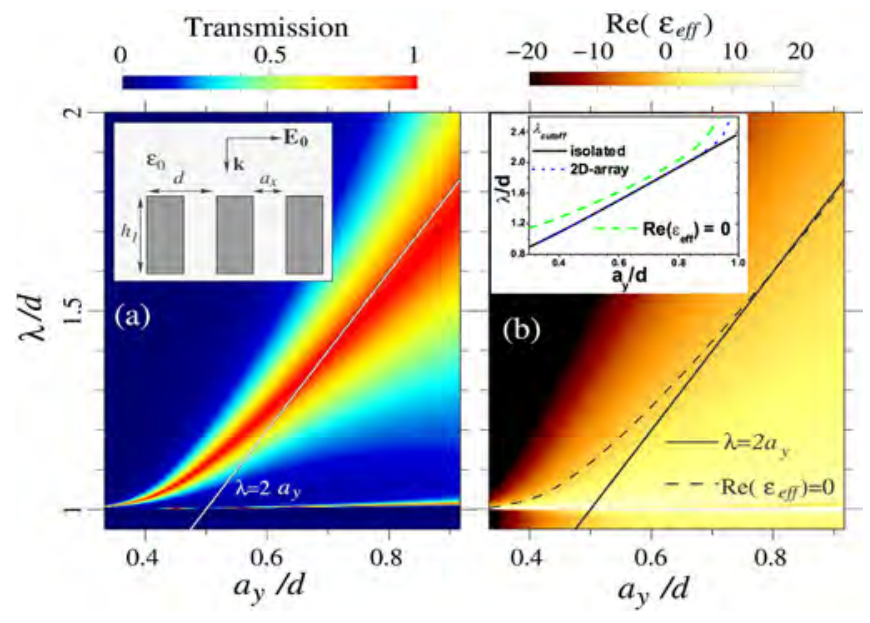

FIG. 1 (color online). (a) Transmittance versus both $a_{y}$ and $\lambda$ for a 2DHA perforated on a PEC film, with geometrical parameters $a_{x}=0.33 d$ and $h_{1}=0.15 d$. Inset contains a schematic picture of the 2DHA. (b) $\operatorname{Re}\left[\epsilon_{\text {eff }}\right]$ as a function of $\lambda$ and $a_{y}$. Solid line: Cutoff wavelength, $\lambda=2 a_{y}$. Dashed line: $\lambda$ that satisfies the condition $\operatorname{Re}\left[\epsilon_{\text {eff }}(\lambda)\right]=0$. In the inset, the corresponding FDTD results for silver 2DHA with the same parameters as in (a) and $d=600 \mathrm{~nm}$ are displayed.

wavelength of the fundamental mode $\left(\mathrm{TE}_{01}\right.$ mode) of the hole waveguide. This resonance appears already in single rectangular holes [16,17] and can be understood as a Fabry-Perot resonance in which the propagation constant inside the hole is zero. From now on, we name cutoff resonance to this hybridized mode that is a combination of the symmetric surface EM mode and the Fabry-Perot resonance. From the knowledge of the zero-order transmission and reflection coefficients, it is possible to retrieve biunivocally the effective optical parameters [18]. The method described in Ref. [18] is reliable provided the zero-order diffracted beams are the only ones propagating in both the reflection and transmission regions (for normal incidence this implies $\lambda>d$ ). The real part of the effective permittivity, $\epsilon_{\text {eff }}$, of the 2DHA structure is shown in Fig. 1(b). Dashed lines and solid lines indicate the wavelength in which $\operatorname{Re}\left[\epsilon_{\text {eff }}\right]=0$ and the cutoff wavelength, respectively. Note that except in the region $\lambda \approx d$, there is a close correspondence between these two wavelengths. In the effective medium approximation $(\lambda \gg d)$, a holey semi-infinite PEC is characterized by a $\operatorname{Re}\left[\epsilon_{\text {eff }}\right]$ that changes from negative $\left(\lambda<\lambda_{C}\right)$ to positive values $(\lambda>$ $\left.\lambda_{C}\right)$, and by a $\mu_{\text {eff }}$ that remains constant and positive [15]. Figure 1(b) shows that the relation between $\lambda_{C}$ and the condition $\operatorname{Re}\left[\epsilon_{\text {eff }}\right]=0$ also holds for a very thin PEC film and for $\lambda$ slightly larger than $d$. Moreover, this link is maintained when moving to frequencies in the optical regime, as shown in the inset of Fig. 1(b). Here the spectral locations of both the cutoff wavelength of the hole waveguide (black line) and the condition $\operatorname{Re}\left[\epsilon_{\text {eff }}\right]=0$ (green dashed line) are rendered as a function of $a_{y}$, for the same geometrical parameters as in Fig. 1(a), and $d=600 \mathrm{~nm}$.
The fact that, both in the PEC and optical regimes, the dielectric response of a holey metal film is mainly controlled by the cutoff wavelength of the hole waveguide is the first important result of this Letter. Notice, however, that for a silver 2DHA in the limit $a_{y} \rightarrow d$, the condition $\operatorname{Re}\left[\epsilon_{\text {eff }}\right]=0$ is linked to the cutoff wavelength calculated with the FDTD method [14] for waveguides forming a 2D periodic array [blue dashed line in the inset of Fig. 1(b)]. In this limit, the cutoff deviates from the one of an isolated waveguide, due to the cross talk between waveguides through the vertical metal walls.

The rest of this Letter is devoted to analyzing the optical properties of the DF structure. The inset of Fig. 2(a) renders the normal incidence transmission spectra for the PEC case. The two transmission peaks previously described for a 2DHA also appear in the DF structure. Figure 2(a) shows the spectral location of the condition $\operatorname{Re}\left[\epsilon_{\text {eff }}\right]=0$ (green dashed line) and the regions in which $\operatorname{Re}\left[\mu_{\text {eff }}\right]$ (red vertical lines) and $\operatorname{Re}\left[n_{\text {eff }}\right]$ (black horizontal lines) are negative. Notice that the cutoff resonance wavelength also controls $\operatorname{Re}\left[\epsilon_{\text {eff }}\right]$ in the case of a PEC-DF structure, as shown in Fig. 2(a). On the other hand, the presence of a second metal layer and a dielectric gap results in the emergence of additional resonant features (transmission peak and dip) in the transmission spectra. Associated with this new resonant behavior, $\operatorname{Re}\left[\mu_{\text {eff }}\right]$ presents negative values [see Fig. 2(a)]. As expected, the effective refractive index is negative when both $\operatorname{Re}\left[\epsilon_{\text {eff }}\right]$ and $\operatorname{Re}\left[\mu_{\text {eff }}\right]$ are negative. This condition is only satisfied when $a_{y}<0.58 d$ (for this set of geometrical parameters). For larger holes, as the magnetic resonance appears at a shorter wavelength than the cutoff peak, the regions of $\operatorname{Re}\left[\mu_{\text {eff }}\right]$ appear where $\operatorname{Re}\left[\epsilon_{\text {eff }}\right]$ is positive, leading to positive values for $\operatorname{Re}\left[n_{\text {eff }}\right]$.

The behavior of the effective optical parameters is very similar for a silver-DF structure. Figure 2(b) shows the same quantities as in Fig. 2(a) but now for a DF structure made of silver with the same geometrical parameters as in Fig. 2(a), and $d=600 \mathrm{~nm}$. As in the PEC case, the link between the condition $\operatorname{Re}\left[\epsilon_{\text {eff }}\right]=0$ and the cutoff wavelength is clearly observed. Moreover, a band of negative $\operatorname{Re}\left[\mu_{\text {eff }}\right]$ presenting a smooth linear dependence with $a_{y}$ also appears. The disappearance of this band for small $a_{y}$ is due to absorption (calculations for a lossless silver show regions of negative $\operatorname{Re}\left[\mu_{\text {eff }}\right]$ for small $a_{y}$ ). Absorption also explains why, as a difference with a PEC-DF, the region of negative $\operatorname{Re}\left[n_{\text {eff }}\right]$ is larger than the one in which both $\operatorname{Re}\left[\epsilon_{\text {eff }}\right]$ and $\operatorname{Re}\left[\mu_{\text {eff }}\right]$ are negative [19]. Notice however that the figure of merit (FOM), defined as the modulus of the ratio between the real and imaginary parts of $n_{\text {eff }}$, is maximum inside the region in which both $\operatorname{Re}\left[\mu_{\text {eff }}\right]$ and $\operatorname{Re}\left[\epsilon_{\text {eff }}\right]$ are negative.

The physical origin of the magnetic resonance described above clearly emerges when looking at the corresponding $E$ field patterns. In Fig. 3 we plot the $E$-field amplitudes for 

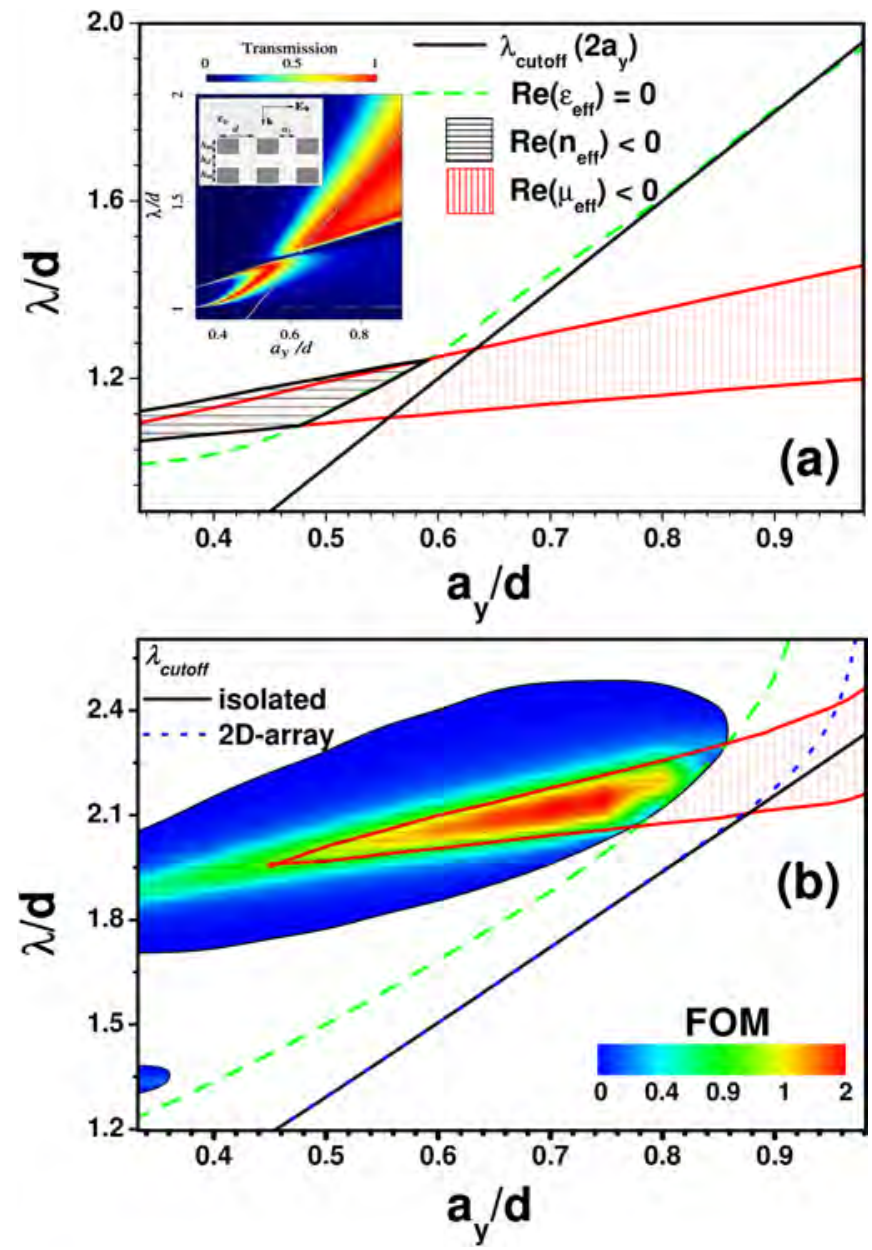

FIG. 2 (color online). (a) The evolution of the spectral locations of both the cutoff wavelength (black line) and the condition $\operatorname{Re}\left[\epsilon_{\text {eff }}\right]=0$ (green dashed line). The regions of negative $\operatorname{Re}\left[\mu_{\text {eff }}\right]$ (red vertical lines) and $\operatorname{Re}\left[n_{\text {eff }}\right]$ (black horizontal lines) are also displayed as a function of $a_{y}$ for the PEC-DF case. Inset: Transmittance versus both $a_{y}$ and $\lambda$ for a PEC-DF structure with the geometrical parameters as in Fig. 1. Solid curve renders $\lambda=2 a_{y}$ line. (b) As in (a), but for a silver-DF structure with $d=600 \mathrm{~nm}$. A contour plot of the FOM is rendered in this panel, for the regions where $\operatorname{Re}\left[n_{\mathrm{eff}}\right]<0$.

the DF structure evaluated at an $x y$ plane between the two metal films [Figs. 3(a) and 3(b)] and at an $x z$ plane that cuts the holes through their centers [Figs. 3(c) and 3(d)]. Figures 3(a) and 3(c) correspond to the PEC-DF case whereas Figs. 3(b) and 3(d) present the results for silver DF. In these calculations, the long edge of the holes is fixed at $a_{y}=0.5 d$ and the $E$ fields are evaluated at the wavelength of the magnetic resonance. In the two frequency ranges analyzed (PEC and optical regimes), the $E$ field is mainly concentrated in the gap region between the metallic films and also has a strong standing wave character in the $x$ direction. The $E$-field intensity maxima are along the ridges of the holes, suggesting that in both cases two SPP-like modes that counterpropagate in the $x$ direction
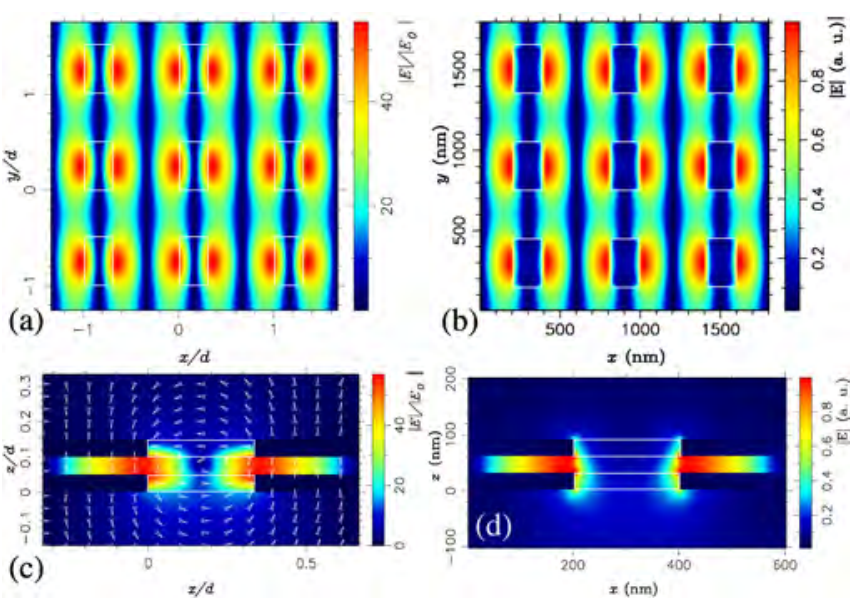

FIG. 3 (color online). (a),(b) The electric field amplitudes at the magnetic resonance evaluated in the $x y$ plane just in the middle of the dielectric slab $(z=0.075 d)$. (c),(d) Show these amplitudes in an $x z$ plane $(y=0.25 d)$. The geometrical parameters are as in Fig. 2 with $a_{y}=0.5 d$. (a),(c) Correspond to the PEC case whereas (b),(d) are for the silver-DF structure with $d=600 \mathrm{~nm}$. White lines mark the positions of the holes.

are involved in the formation of the resonance. A dielectric gap placed between two metallic films supports the propagation of SP modes that are usually called gap SPPs [20]. For two nonperforated silver films of thickness $h_{m}=$ $30 \mathrm{~nm}$ and separated by a $30 \mathrm{~nm}$ vacuum gap (geometrical parameters of the silver-DF structure), the spectral location of the gap-SPP mode that could be excited by a normal incident plane wave can be calculated by evaluating the gap-SPP dispersion relation at $k_{x}=2 \pi / d$. This calculation leads to $\lambda_{\text {res }}=1050 \mathrm{~nm}=1.75 d$, that nicely coincides with the limit $a_{y} \rightarrow 0$ of the magnetic resonance leading to negative $n_{\text {eff }}$ displayed in Fig. 2(b). Folding of the gap-SPP dispersion relation at $k_{x}=4 \pi / d$ originates the small NRI region located at $\lambda \approx 1.3 d$ for small $a_{y}$ observed in Fig. 2(b). When the metal is a PEC, gap surface EM modes that are very similar to the gap-SPP modes in the optical regime are created due to the presence of a hole array drilled in the PEC films, even though nonperforated PEC films do not support the propagation of gap-SPP modes. The dispersion relation of these geometry-induced gap-SPP modes lies very close to the light line, explaining why the magnetic resonance band for a PEC-DF appears very close to $\lambda=d$ [see Fig. 2(a)]. The connection between the resonant magnetic response and the excitation of gap-SPP modes is highlighted for the PEC case in Fig. 3(c). The electric field is pointing at opposite directions at the two sides of the dielectric slab, generating a displacement current that resembles that of the one created by a magnetic dipole parallel to the $y$ direction. This is the standard explanation of the magnetic behavior in DF structures, as described in Ref. [4]. The second important result of this Letter is, however, the existence of a link between the 


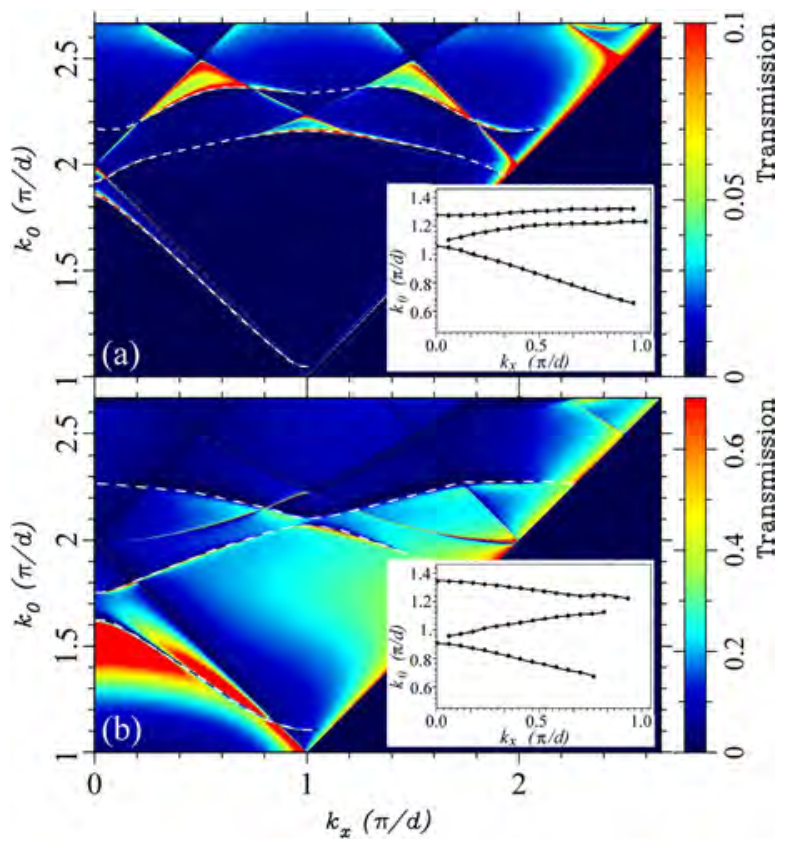

FIG. 4 (color online). Transmittance as a function of both incident wave number $\left(k_{0}\right)$ and parallel momentum $\left(k_{x}\right)$ for $a_{y}=a_{x}=0.33 d$ (a) and $a_{y}=2 a_{x}=0.66 d$ (b). White dashed lines show the spectral locations of the gap-SPP modes in the holey structure. Insets: Corresponding dispersion relations for the gap-SPP modes in DF structures made of silver with $d=$ $600 \mathrm{~nm}$.

resonant magnetic response of a DF structure with the excitation of gap-SPP modes, both in the PEC and optical regimes.

If gap-SPP modes are involved in the resonant magnetic response, it is expected that this response will be very sensitive to the angle of incidence. This is demonstrated in the main panels of Fig. 4 that renders contour plots of the transmittance versus wave number $\left(k_{0}=2 \pi / \lambda\right)$ and parallel momentum $\left(k_{x}\right)$ for two different PEC-DF structures. In Fig. 4(a), $a_{y}=0.33 d$ whereas Fig. 4(b) shows the case $a_{y}=0.66 d$. The spectral locations of the transmission resonances due to the excitation of gap-SPP-like modes (leading to negative $\mu_{\text {eff }}$ ) are underlined with white dashed lines. Because of the folding of the dispersion curves of the gap-SPP-like modes inside the first Brillouin zone, there are three branches that present resonant magnetic response. For small holes, these curves highly disperse with $k_{x}$ (angle of incidence), specially the lower branch. As $a_{y}$ is increased [see Fig. 4(b)], the hybridization of the gap-SPPlike modes with the cutoff resonance decreases the dispersion of the gap-SPP-like modes with $k_{x}$. Insets in Fig. 4 show the corresponding results for the silver-DF structures. The locations of the optical gap-SPP modes (and their associated NRI) also disperse with parallel momentum, although this dispersion is less pronounced than the one found in the PEC case. The fact that, both in the PEC and optical limits, the resonant magnetic response (and the corresponding NRI) in a DF structure disperses with the angle of incidence is the third main finding of our work.

In conclusion, we have presented a complete theory of the NRI response of double-fishnet structures by analyzing two very distinct frequency regimes. Our results show that these structures can be interpreted as holey plasmonic metamaterials. Their electric permittivity is governed by the cutoff frequency of the hole waveguide. Negative values of the magnetic permeability are associated with the excitation of gap-SPP modes in the dielectric film. We expect that this clear identification of the common physical mechanisms behind the NRI response in the PEC and optical regimes will lead to improvements in the design of NRI metamaterials.

Financial support by the EU under Contract No. ISTFP6-034506 and Spanish MEC under Contract No. MAT2005-06608-C02 and Nanolight.es is gratefully acknowledged.

*Corresponding author.

fj.garcia@uam.es

[1] T. W. Ebbesen et al., Nature (London) 391, 667 (1998).

[2] S. Zhang et al., Phys. Rev. Lett. 95, 137404 (2005).

[3] S. Zhang et al., Opt. Express 13, 4922 (2005).

[4] For a recent review on negative-index metamaterials, see V. M. Shalaev, Nat. Photon. 1, 41 (2007).

[5] C. M. Soukoulis, S. Linden, and M. Wegener, Science 315, 47 (2007).

[6] G. Dolling et al., Appl. Phys. Lett.89, 231118 (2006).

[7] G. Dolling et al., Science 312, 892 (2006).

[8] M. Beruete, M. Sorolla, and I. Campillo, Opt. Express 14, 5445 (2006).

[9] M. Kafesaki et al., Phys. Rev. B 75, 235114 (2007).

[10] J. B. Pendry, Phys. Rev. Lett. 85, 3966 (2000).

[11] Handbook of Optical Constants of Solids, edited by E. D. Palik (Academic, New York, 1985).

[12] L. Martín-Moreno et al., Phys. Rev. Lett. 86, 1114 (2001).

[13] J. Bravo-Abad, F. J. García-Vidal, and L. Martín-Moreno, Phys. Rev. Lett. 93, 227401 (2004).

[14] A. Taflove and S.C. Hagness, Computational Electrodynamics: The Finite-Difference Time-Domain Method (Artech House, Boston, 2005).

[15] J. B. Pendry, L. Martín-Moreno, and F. J. García-Vidal, Science 305, 847 (2004).

[16] F. J. García-Vidal et al., Phys. Rev. Lett. 95, 103901 (2005).

[17] Z. Ruan and M. Qiu, Phys. Rev. Lett. 96, 233901 (2006).

[18] D. R. Smith et al., Phys. Rev. B 65, 195104 (2002).

[19] The general condition for having $\operatorname{Re}\left[n_{\text {eff }}\right]<0$ is $\operatorname{Re}\left[\epsilon_{\text {eff }}\right]\left|\mu_{\text {eff }}\right|+\operatorname{Re}\left[\mu_{\text {eff }}\right]\left|\epsilon_{\text {eff }}\right|<0$. For more details, see R. A. Depine and A. A. Lakhtakia, Microw. Opt. Technol. Lett. 41, 315 (2004).

[20] E. N. Economou, Phys. Rev. 182, 539 (1969). 